\title{
Experimental Evaluation of an On-line RSA Algorithm for SDN-Controlled Optical Metro Networks with VCSEL-based S-BVTs
}

\author{
R. Martínez, R. Casellas, M. Svaluto Moreolo, J. M. Fabrega, R. Vilalta, R. Muñoz and Laia Nadal \\ Centre Tecnològic de Telecomunicacions de Catalunya (CERCA/CTTC) \\ Castelldefels, Spain \\ rmartinez@cttc.es
}

\author{
V. López and J. P. Fernández-Palacios \\ Telefónica $I+D$, GTCO \\ Madrid, Spain
}

\author{
D. Larrabeiti and G. Otero \\ Universidad Carlos III \\ Madrid, Spain
}

\begin{abstract}
Upcoming metro networks are required to transport huge and highly dynamic data traffic whilst resulting cost and power efficient. Thus, novel metro network architectures are being investigated to increase the transport capacity (e.g., leveraging dense photonic integrated technologies), and provide effective SDN-based network programmability. The EU-H2020 PASSION project targets such a metro network design exploiting: i) programmable, modular, cost-efficient and low-power sliceable bandwidth variable transceivers (S-BVTs) formed by a pool of vertical cavity surface emitting lasers (VCSEL) and Coherent Receivers (CO-Rx); ii) hierarchical switching nodes in a flexigrid network to attain all-optical transport and aggregation. The SDN-based control functions considers the features of the adopted PASSION transport layer. First, we detail the defined interfaces (APIs) for the automatic transport network programmability. Such (RESTful-based) interfaces are validated within the ADRENALINE testbed. Next, upon dynamic connection requests with heterogenous data rate demands, an on-line Routing and Spectrum Assignment (RSA) algorithm based on a modified $K$ shortest path (K-SP) is proposed. To accommodate a connection request, a set of optical flows having their own $S$ BVT VCSEL and CO-Rx devices at the endpoints and path's frequency slots are selected and configured. Each optical flow is subject to the spectral continuity and contiguity constraints. The performance of the RSA algorithm for several traffic loads is experimentally evaluated through different figures of merit (e.g., blocked bandwidth ratio, average used of S-BVT devices, etc.) and diverse RSA's K-SP settings.

Index Terms-SDN, Flexi-Grid Metro Networks, S-BVT
\end{abstract}

\section{INTRODUCTION}

Metro networks are crucial to cope with the huge transport and highly dynamic traffic of $5 \mathrm{G}$ (and beyond) network services. A CISCO report states that $33 \%$ of the overall service provider network capacity will be required within the metro network segment [1]. Thus, such networks need to be designed with enhanced flexibility and higher capacity while attaining

The research leading to these results has received funding from the EUH2020 PASSION Project (780326) and by Spanish MICINN AURORAS (RTI2018-099178-B-I00) project. reduced cost, energy and footprint [2]. The European H2020 PASSION project [5] targets those metro network challenges exploiting the advantages of photonic technologies over flexigrid networks [6]. Specifically, dense photonic integration and cost-effective photonic devices are adopted to deploy programmable and flexible network elements such as optical transceivers.

Network programmability is done by a centralized SoftwareDefined Networking (SDN) controller. This entails automatic functions and interfaces to: i) dynamically compute paths and select optical resources; ii) configure the network elements (i.e., transceivers and optical switches) to accommodate the requests. To do this, the controller relies on defined interfaces (APIs) communicating (e.g., for network configuration purposes) with the agents locally managing every underlying network element.

In the PASSION project, the emerging photonic technology of vertical cavity surface emitting laser (VCSEL) at longwavelength is considered to deploy a novel, low cost, power and footprint SDN-controlled transceivers [2] [3]. VCSEL technology and photonic integrated circuits (PICs) are combined to attain a modular and scalable (approaching a pay-asyou-grow strategy) sliceable bandwidth variable transceivers (S-BVTs) reaching Tb/s capacity within the metro networks. The modular S-BVT is based on a hierarchical architecture formed by the tuple Module/subModule/VCSELs [4]. A Module is bound to a silicon-on-insulator (SOI) chip. Such a Module integrates 4 subModules having 10 VCSELs each. Thus, a S-BVT with a single Module (i.e., SOI) where all the possible VCSELs are licensed achieves a compact transmitter (Tx) with $2 \mathrm{~Tb} / \mathrm{s}$ maximum capacity. Each $20 \mathrm{GHz}-$ bandwidth VCSEL is directly modulated with adaptive multicarrier modulation formats (either DMT or OFDM) providing up to $50 \mathrm{~Gb} / \mathrm{s}$. Individual VCSEL device operates at a particular wavelength in the C-band ranging the spectrum between $191.900-195.875$ THz. The modular S-BVT can achieve higher transport ca- 
pacity ( $\geq 2 \mathrm{~Tb} / \mathrm{s}$ ) by, for instance, adding (up to 4) SOI-chip fundamental Modules. This results on a fully-equipped S-BVT providing a maximum transmission of $8 \mathrm{~Tb} / \mathrm{s}$ via $160 \mathrm{VCSELs}$ whose optical carriers are spaced $25 \mathrm{GHz}$. The advantage of this modular S-BVT is to add both Modules (SOI chips) and licensed VCSELs as needed to cope with the envisaged traffic needs. At the S-BVT Receiver (Rx), a set of integrated Coherent Receivers (CO-Rxs) with tunable local oscillators are used.

The considered metro optical network is formed by heterogenous optical switches. The purpose is twofold: providing end-to-end all-optical transport; and effective optical layer aggregation. The heterogenous optical switches are associated to a defined Hierarchical Level (HL): HL4, HL3 and HL2/1. The HL determines the optical switch capacities such as the capability to originate/terminate optical flows and/or all-optical routing and aggregation of different flows. Specifically, HL4 nodes border the access network segment (e.g., mobile infrastructure). These nodes are equipped with S-BVTs configuring a limited number of licensed VCSELs and CO-Rxs devices to offer capacities up to e.g., $1 \mathrm{~Tb} / \mathrm{s}$. HL3 nodes, on the other hand, are used as transit optical switches for all-optically interconnecting HL4 and HL2/1 nodes. Thus, HL3 nodes do not integrate S-BVT elements. Finally, HL2/1 nodes connect to the core network, and are attached to fully-equipped S-BVTs (i.e., up to $8 \mathrm{~Tb} / \mathrm{s}$ of aggregated transport capacity).

In the scope of the PASSION metro network, the motivation of this work is to identify the requirements, functions and operations to enable an SDN controller dynamically computing and setting up heterogenous data rate optical connections. To do that, new control interfaces (APIs) are defined for the network programmability. Additionally, to compute end-to-end paths and select the resources, on-line Routing and Spectrum Assignment (RSA) algorithms are triggered considering the technological features of the PASSION transport layer. In this regard, for instance, the S-BVT VCSEL transmission capacity depends on the total distance and number of hops of the computed spatial path (i.e., node and links). The resulting net data rate achieved at the destination node may be lower than the maximum achievable by the VCSEL (i.e., $50 \mathrm{~Gb} / \mathrm{s}$ ) due to the accumulated optical physical impairments. In light of this, it is considered that S-BVT VCSELs perform on different Operation Modes bound to diverse different data rates. Therefore, to satisfy the requested bandwidth of an optical connection, a number $(\geq 1)$ of optical flows are generated and set up. The goal is that the individual data rates provided by each optical flow are aggregated to fulfil the targeted connection bandwidth requirements. Each optical flow allocates its own S-BVT VCSEL and CO-Rx devices (at the endpoints) as well as the spectral resources (i.e., frequency slot, FS) on each link along the path. The selected FS is subjected to the spectrum continuity and contiguity constraints.

Herein, a novel RSA algorithm is devised for the adopted underlying PASSION network infrastructure. The output of the RSA algorithm is formed by: i) the selected S-BVT VCSELs at the source node; ii) the CO-Rxs (with their tuned local oscillators) at the destination node; and iii) the optical spectrum (i.e., FS) for each required optical flow. The RSA algorithm is based on a modified $K$ shortest path (K-SP) called co-routed optical flows (RSA-CR). In short, for a given connection request, the RSA-CR algorithm first determines the number of required optical flows that are then established over the same spatial path but occupying different spectral resources, i.e., S-BVT VCSELs, CO-Rx and FSs.

This work provides the actual validation of the designed control interfaces (APIs) to program the targeted PASSION transport network. The devised RSA-CR, upon different the $\mathrm{K}-\mathrm{SP}$ computations, is experimentally evaluated within the CTTC ADRENALINE testbed under dynamic traffic load. The numerical results are presented and discussed in terms of several figures of merit: the blocked bandwidth ratio (BBR), the average number of used S-BVTs VCSELs and CO-Rxs and the average connection setup time.

\section{SDN-CONTROLled OpticAl Metro Network}

Fig. 1 depicts an example of a PASSION SDN-controlled flexi-grid optical metro network. The Connection Request Application requests (via a RESTful API) the SDN controller to dynamically set up optical connections. The computed path and selected resources are then configured also via a defined RESTful API (see Section IV-A). The description of the defined RESTful APIs are detailed below in section IV-A.

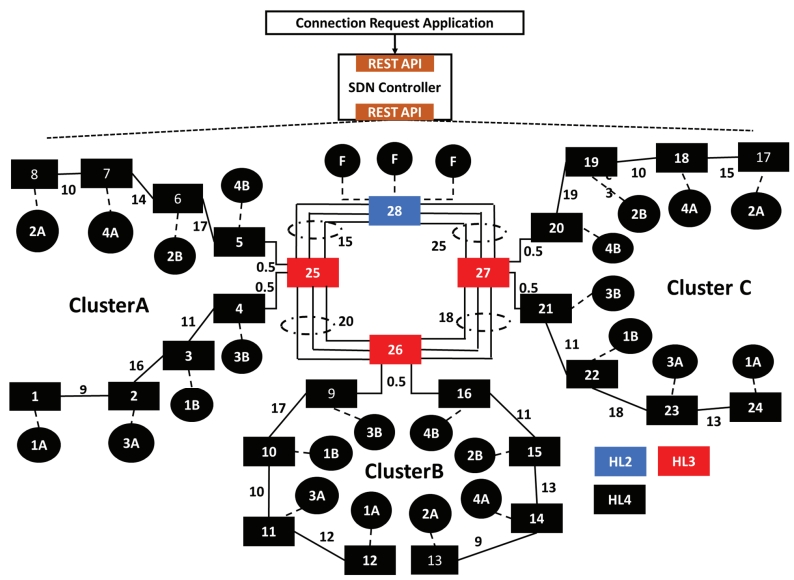

Fig. 1: SDN-controller Optical Metro Network Scenario.

The considered metro network follows a star-ring topology formed by three identical clusters: A, B and C. Each cluster has a pair of linear networks (branches) interconnected through a ring network. The metro optical switches are associated to one of the defined HL types. HL4 nodes (represented by black squares in Fig. 1) provide traffic aggregation to the access network segment. This is done via an S-BVT (represented by a circle in Fig. 1). Those S-BVT devices are configured by a single Module (i.e., SOI) with 20 licensed VCSELs offering a transport capacity of up to $1 \mathrm{~Tb} / \mathrm{s}$. Additionally, 20 CO-Rxs are equipped at each HL4 S-BVT Rx. As said, S-BVT VCSELs operate at a particular optical carrier in the spectral range between $191.900-195.875 \mathrm{THz}$. 
Therefore, to support in a cluster all the frequencies for the targeted spectral range, each branch deploys 4 HL4 nodes. The optical carriers in the 4 resulting S-BVTs per branch are spaced $50 \mathrm{GHz}$. The above is reflected in Table I, where clusters' S-BVTs are labeled by 1A, 1B, 2A, ..., 4B. Moreover, observe that in the whole cluster (encompassing 2 branches) deployed S-BVT's optical carriers are spectrally spaced 25 GHz. The specific assignment of the S-BVT VCSELs' optical carriers (within a cluster) is conveniently realized to avoid spectral collisions when filtering on the traversed HL4 optical switches. The reason behind this is that HL4 nodes are built using Array Wavelength Grating (AWG) of $50 \mathrm{GHz}$.

HL3 nodes, represented by red squares in Fig. 1, are the transit optical switches with $25 \mathrm{GHz}$ filters using Wavelength Selective Switches (WSS). HL3 nodes provides all-optical aggregation and routing, and thus no S-BVTs are placed. Finally, HL2/1 nodes (blue squares in Fig. 1) are also based on $25 \mathrm{GHz}$ WSS filters. The HL2/1 node are attached to 3 fully-equipped S-BVTs, each having 160 VCSELs and 160 CO-Rxs. Fully-equipped S-BVT implements all the $(25 \mathrm{GHz}-$ spaced) optical carriers within the targeted spectral range, thus reaching a transport capacity up to $8 \mathrm{~Tb} / \mathrm{s}$.

TABLE I: S-BVTs with supported VCSELs optical carriers

\begin{tabular}{|c|c|c|}
\hline S-BVT & $\begin{array}{c}\text { No. VCSELs } \\
\text { \& CO-Rxs }\end{array}$ & Supported Frequencies (THz) \\
\hline 1A & 20 & $191.900,192.100 .192 .300, \ldots, 195.700$ \\
\hline 1B & 20 & $192.000,192.200 .192 .400, \ldots, 195.800$ \\
\hline 2A & 20 & $191.925,192.125,192.325, \ldots, 195.725$ \\
\hline 2B & 20 & $192.025,192.225,192.425, \ldots, 195.825$ \\
\hline 3A & 20 & $192.950,192.150,192.350, \ldots, 195.750$ \\
\hline 3B & 20 & $192.050,192.250,192.450, \ldots, 195.850$ \\
\hline 4A & 20 & $192.975,192.175,192.375, \ldots, 195.775$ \\
\hline 4B & 20 & $192.075,192.275,192.475, \ldots, 195.875$ \\
\hline F & 160 & $191.900,191.925,191.950, \ldots, 195.875$ \\
\hline
\end{tabular}

\section{RSA-CR ALGORITHM: DESCRIPTION}

Connection requests (req) are defined by the source node $(s r c)$, the destination node $(d s t)$ and the bandwidth $(b w)$ in $\mathrm{Gb} / \mathrm{s}$. The RSA algorithm seeks for a feasible route formed by: i) the src's S-BVT Tx resources (i.e., VCSELs); ii) the $d s t$ 's SBVT Rx resources (i.e., CO-RXs with the central frequency at each LO); iii) the spatial and the spectral (i.e., FS) path. A FS is determined using recommendation ITU-T G.694.1 [6]. This defines a FS by the nominal central frequency $(n)$ and the slot width $(m)$. The nominal central frequency (NCF) granularity is $6.25 \mathrm{GHz}$. For a given FS, the central frequency (in $\mathrm{THz}$ ) is determined by $193.100 \mathrm{THz}+n * 0.00625 \mathrm{THz}$ (being $n$ either a positive or negative integer); the FS slot width (in $\mathrm{GHz}$ ) is obtained by $0.00625^{*} m$ ( $m$ is a positive integer).

To set up a req, it is likely that a number (Num) of optical flows, each allocating their own S-BVT VCSEL and CO-Rx devices and FS $(n, m)$ are set up. The purpose is that the aggregated data rate of the Num optical flows satisfies the req's $b w$. As commented above, this depends on the spatial path (distance and number of hops) because of the net VCSEL data rate at the $d s t$ may be lower than the maximum VCSEL transmission rate. We consider that S-BVT VCSELs operate into three operational modes $(O M)$, namely high, medium and low. Each $O M$ regards to a data rate for a maximum path distance and number of hops as shown in Table II. At the time being, exhaustive data plane experiments are being conducted in the PASSION project to derive accurate values for the Table II. That said, and without loss of generality, the RSA algorithm aims at selecting the $O M$ providing the higher VCSEL data rate as long as the distance and number of hops restrictions are satisfied. This does reduce the required Num of optical flows for a req, which in turn yields a more efficient use of the network resources.

TABLE II: VCSEL operational modes

\begin{tabular}{|c|c|c|c|}
\hline $\begin{array}{c}\text { VCSEL Mode } \\
\text { (OM) }\end{array}$ & data rate (Gb/s) & $\begin{array}{c}\text { Max. Path } \\
\text { Distance }(\mathbf{k m})\end{array}$ & $\begin{array}{c}\text { Max. Number } \\
\text { of Hops }\end{array}$ \\
\hline high & 50 & 30 & 5 \\
\hline medium & 40 & 75 & 9 \\
\hline low & 25 & 150 & 15 \\
\hline
\end{tabular}

The inputs used by the RSA algorithm are: i) the src SBVT Tx (VCSEL) availability and their optical carriers; ii) the dst S-BVT Rx (CO-Rx) availability; iii) the network topology and link attributes (i.e., cost, length and available NCFs). To discuss the RSA algorithm, the following notation is used:

\begin{tabular}{|c|c|}
\hline req & Optical connection request. \\
\hline$s r c, d s t$ & The source and destination nodes for req. \\
\hline$b w$ & Bit rate (in $\mathrm{Gb} / \mathrm{s}$ ) for $r e q$. \\
\hline$O M$ & VCSEL operation modes sorted by data rate. \\
\hline$O M[i] . r$ & Data rate (in $\mathrm{Gb} / \mathrm{s})$ for $O M[i]$ \\
\hline$O M[i] . d$ & Maximum distance (in $\mathrm{km}$ ) for $O M[i]$. \\
\hline$O M[i] \cdot h$ & Maximum number of hops for $O M[i]$ \\
\hline Num & $\begin{array}{l}\text { Number of VCSELs and CO-Rxs for } O M[i] \text {; computed } \\
\text { by }[b w / O M[i] . r] \text {. }\end{array}$ \\
\hline vcsel $_{n}$ & Number of unused VCSELs at $n$ \\
\hline vcsel $_{n} \cdot N C F$ & Unused NCFs on available VCSELs at $n$. \\
\hline $\operatorname{coR} x_{n}$ & Number of unused CO-Rxs at $n$. \\
\hline $\operatorname{coR} x_{n} \cdot N C F$ & Used NCFs by the occupied CO-Rxs at $n$. \\
\hline$A$ & $\begin{array}{l}K \text { Shortest paths (SPs) between } s r c \text { and } d s t \text { sorted by } \\
\text { distance and hops. }\end{array}$ \\
\hline$A[k] . d$ & Distance (in $\mathrm{km}$ ) of the $k^{t h} \mathrm{SP}$. \\
\hline$A[k] \cdot h$ & Number of hops of the $k^{t h} \mathrm{SP}$. \\
\hline$A[k] . N C F$ & Unused and common NCFs over $k^{t h} \mathrm{SP}$. \\
\hline$A[k] . \operatorname{set} N C F$ & $\begin{array}{l}\text { Unused and common NCFs; computed intesecting } \\
A[k] . N C F \cap v_{\text {csel }} \text { src. } N C F \cap \frac{\mathrm{coR} x_{d s t} . N C F}{} \text {. }\end{array}$ \\
\hline$F S$ & Optical flow FS $(n, m)$ \\
\hline findFS & Returns the first FS over $A[k]$. set $N C F$. \\
\hline$p_{l}$ & Path $l$ for the optical flow $(0 \leq l<N u m)$. \\
\hline$P$ & Set of Num ps for the optical flows. \\
\hline & spatial route for $p_{l}$ \\
\hline & $(n, m)$ for $p_{l}$ \\
\hline
\end{tabular}

The objective function of the RSA-CR algorithm is to set up every incoming req satisfying the set of spectral constraints while attaining an efficient use of all the network resources. The RSA-CR is based on a modified K-SP mechanism returning the first $k t h$-SP that accommodates the Num optical flows for a req. Fig. 2 depicts an example of the RSA-CR algorithm. A req of $100 \mathrm{~Gb} / \mathrm{s}$ between S-BVT Tx at node 9 and S-BVT Rx at node 28 arrives. Using the high OM, two VCSELs (at $50 \mathrm{~Gb} / \mathrm{s}$ ) and two CO-Rxs are needed. This entails allocating two optical flows (i.e., X and Y). Optical flow X occupies the S-BVT's VCSEL associated to the optical carrier at $192.050 \mathrm{THz}$, whilst optical flow Y allocates the VCSEL with the optical carrier at $192.250 \mathrm{THz}$. At the dst, S-BVT CO-Rx LOs are programmed to receive such optical 
carriers. Both $\mathrm{X}$ and $\mathrm{Y}$ optical flows have their own FSs (i.e., $n, m)$. The central frequency of the FS $(n)$ is determined by the selected VCSEL optical carrier. On the other hand, the FS slot width $(m)$ is adjusted according to the filtering features of each traversed HL node. For instance, at node 9 (HL4), the FS of the optical flow $\mathrm{X}$ occupies $n=-168$ (ITU-T channel for $192.050 \mathrm{THz}$ ) and $m=4$ (slot width of $50 \mathrm{GHz}$ ). For the optical flow Y, at the same node, the FS is $n=-136$ and $m=4$. However, at HL3 nodes (26 and 25) and HL2/1 node (28) with $25 \mathrm{GHz}$ filters, the FS for optical flow $\mathrm{X}$ uses $n=-166$ and $m=2$. Likewise, for the optical flow $\mathrm{Y}$, the FS in those nodes is $n=-134$ and $m=2$. It is worth mentioning that although optical flow's FS parameters ( $n$ and $m$ ) may vary along the path, the optical spectrum carrying the effective data (i.e., within $25 \mathrm{GHz}$ ) remains the same.

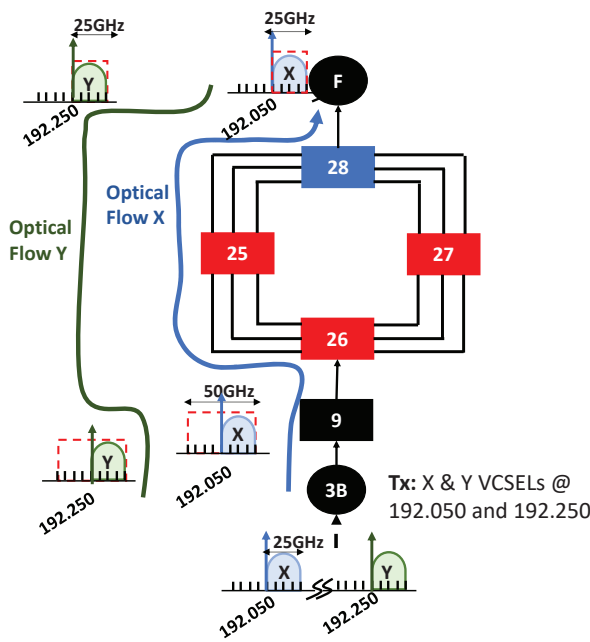

Fig. 2: Example of RSA-CR algorithm.

The pseudocode of the RSA-CR is shown in Algorithm 1. Upon receiving a req, the RSA computes the $A$ set with K-SPs between the $s r c$ and the $d s t$ nodes. The $K$ routes are sorted by the distance and number of hops. Then, the RSA iterates through the VCSELs' $O M$ s starting from the high mode until a feasible solution is found. If all $O M \mathrm{~s}$ are checked and no solution is obtained, the req is blocked (i.e., NoPath).

For each explored $O M_{i}$, the Num of optical flows is computed as the higher integer above $b w / O M_{i} . r$. Then, at least Num VCSELs and CO-Rxs at both S-BVT Tx and Rx need to be available. If not, the req is blocked. Conversely, the pre-computed $A$ set of K-SPs are checked. The first $A[k]$ path satisfying the set of constraints becomes the path solution. To this end, for an $A[k]$ path, it is checked that both the distance and number of hops do not exceed the maximum permitted by the considered $O M_{i}$. If this occurs, $A[k]$ path is discarded. Otherwise, the set of unused and common NCFs (i.e., $A[k] . \operatorname{set} N C F$ ) is computed. This is done intersecting the available NCFs along the ${ }^{k} t h$-SP path links, the associated NCFs of each available S-BVT VCSEL, and those NCFs not being occupied by the used S-BVT CO-Rxs at the dst. The resulting $A[k]$.set $N C F$ is the input of the findF $S$ function. This function returns the first available $F S$ in $A[k] . s e t N C F$. The found $F S$ is associated to the path ( $p$ ) for one of the Num optical flows to be computed. Next, $p$ is appended to the targeted $P$ set. The spectral resources (i.e., NCFs) used in the $F S$ for previously computed $p$ are removed from $A[k]$.set $N C F$. This allows the RSA algorithm not considering already booked NCFs for the remaining optical flows to be computed. Finally, if $P$ reaches the required $N u m$ of optical flows, the RSA-CR successes. Otherwise, $A[k]$ path is discarded and the next $A[k+1]$ is checked. If none of the $A$ paths succeeds, next $O M_{i}$ is explored.

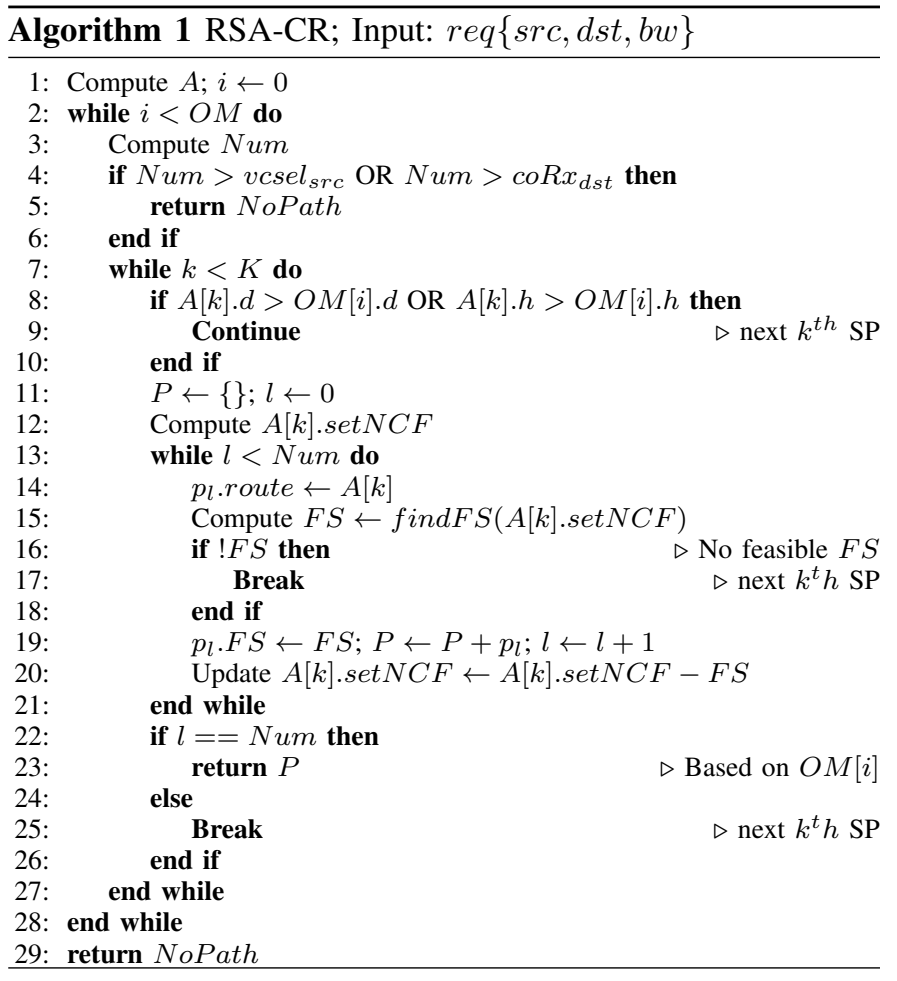

\section{EXPERIMENTAL VALIDATION AND EVALUATION}

The validation and performance evaluation of the SDNcontrolled metro network and devised RSA-CR algorithm are realized at the control plane level within the CTTC ADRENALINE testbed [7]. The PASSION metro network shown in Fig. 1 is deployed over 28 Linux-based servers. Each server hosts and runs an HLx node agent which logically emulates the (de-)allocation of optical flows. Servers running HL4 agents (i.e., nodes 1 to 24 in Fig. 1) also host the agents for controlling S-BVT devices configured as described in Table I. The server for the node 28 agent (i.e., HL2/HL1) co-locates three fully-equipped S-BVT agents. Finally, both the Connection Request Application and SDN controller run in separated servers.

\section{A. REST API-based SDN Control Validation}

The SDN controller operations (i.e., computation and programmability) triggered upon receiving a req are executed according to a defined workflow. Such a workflow is supported through two control interfaces (APIs) that allow: 
- Requesting/deleting reqs between the Connection Request Application and the SDN controller.

- Programming S-BVT (i.e., Tx and Rx) devices between the controller and the transceivers agents.

- Configuring HLx nodes (i.e., cross-connections) between the controller and the node agents.

The following provides the validation of the implemented control workflow and defined RESTful APIs. At step 1 of Fig. 3a a req arrives to the SDN controller via a POST / Isp message from the Connection Request Application. This specifies the $s r c$, the $d s t$ and the $b w$. Next, the RSA computation is triggered. To do that, the SDN controller first retrieves the status of both the VCSELs and the CO-Rxs at the S-BVT endpoints (step 2). This is done sending to the corresponding S-BVTs' agents both the GET / sbvtTx and GET / sbvtRx commands. Then, if the RSA-CR succeeds, the computed output path provides the optical resources to be configured (steps 3 and 4). For the S-BVT Tx configuration (step 3), the controller relies on the POST/sbvtRx/freqSlot command. The payload of this message carries a set of (JSONencoded) parameters (shown in Fig. 4a) describing the central frequency/carrier $(n)$ of the selected VCSEL and the slot width $(m)$. Analogously, the S-BVT Rx is also programmed via the POST / sbvtRx/freqslot. The contents of this message determines the LO to be tuned (i.e., $n$ ) at the chosen available CO-Rx element (see Fig. 4b) .

HL node cross-connections (step 4) are programmed via the POST / opticalSwitch/connections command. The message contents provides the spatial and spectral switching information to be configured: portIn, portOut and $F S(n, m)$. Those (JSON-encoded) contents are shown in Fig. 4c. Once all the optical resources configured, the SDN controller responds to the Connection Request Application (step 5). For the sake of completeness, Fig. $3 \mathrm{~b}$ provides the experimental validation of the described workflow and control APIs.

\section{B. Numerical Results}

The performance evaluation of the RSA-CR algorithm is done under dynamic connection requests with different data rates $(b w)$. Requests arrive according to a Poisson process with mean inter-arrival time set to $5 \mathrm{~s}$. The connection duration (i.e., holding time, HT) is exponentially modeled whose mean is varied to obtain different traffic loads. The $s r c$ and $d s t$ nodes of each req are randomly selected for unidirectional connections between the HL4 nodes and the core (HL2) optical switch (i.e., node 28), and viceversa. The $b w$ is uniformly distributed as multiple of $50 \mathrm{~Gb} / \mathrm{s}$ up to $200 \mathrm{~Gb} / \mathrm{s}$. Each result data point is obtained generating $10 \mathrm{k}$ connection requests.

Network links are formed by bidirectional optical fibers. Each fiber support 644 NCFs spaced $6.25 \mathrm{GHz}$ to span 191.900 - $195.875 \mathrm{THz}$ spectrum range. The link length (in $\mathrm{km}$ ) is shown in Fig. 1. This allows computing the distance of candidate paths being essential to the RSA for choosing the most suitable VCSEL's $O M$ (see Table II). The evaluation compares, for different traffic loads, how the RSA-CR algorithm performs varying the $K$ value: $1,3,6$ and 9. The figures of merit are the BBR, the average number of used S-BVTs VCSELs and CO-Rxs, and the average connection setup time. The BBR provides the ratio between the blocked bandwidth and the total requested bandwidth. Thereby, the lower the $\mathrm{BBR}$, the better an RSA algorithm performs.

TABLE III: S-BVTs with supported VCSELs optical carriers

\begin{tabular}{|c|c|c|c|c|c|c|c|c|}
\hline RSA-CR (K SPs) & \multicolumn{2}{|c|}{$\mathbf{1}$} & \multicolumn{2}{|c|}{$\mathbf{3}$} & \multicolumn{2}{|c|}{$\mathbf{6}$} & \multicolumn{2}{|c|}{$\mathbf{9}$} \\
\hline HT (s) & $\mathbf{4 0 0}$ & $\mathbf{1 2 0 0}$ & $\mathbf{4 0 0}$ & $\mathbf{1 2 0 0}$ & $\mathbf{4 0 0}$ & $\mathbf{1 2 0 0}$ & $\mathbf{4 0 0}$ & $\mathbf{1 2 0 0}$ \\
\hline $\begin{array}{c}\text { Av. Used } \\
\text { VCSEL }\end{array}$ & 10.2 & 16.1 & 10.6 & 18.7 & 10.7 & 18.9 & 10.6 & 19.2 \\
\hline $\begin{array}{c}\text { Av. Used } \\
\text { CO-Rx }\end{array}$ & 10.2 & 16.3 & 10.6 & 18.7 & 10.8 & 19.0 & 10.6 & 19.2 \\
\hline $\begin{array}{c}\text { Av. Setup } \\
\text { Time (ms) }\end{array}$ & 83.5 & 79.7 & 83.6 & 81.7 & 85.0 & 83.7 & 88.6 & 87.1 \\
\hline
\end{tabular}

Regardless of the $K$ value, as HT increases (i.e., traffic load grows) the BBR performance is worsened as shown in Fig. 5. Obviously, more optical flows occupying resources (i.e., S-BVT VCSELs and CO-Rxs, links's NCFs) co-exist. This makes the RSA algorithm finding more problems to provide a feasible path and $F S$ for each req's optical flow. Two reasons do cause the RSA algorithm to fail (i.e., NoPath): i) no sufficient available S-BVT resources at either $s r c$ or $d s t$ for the Num optical flows; ii) unable to guarantee the end-to-end spectrum contiguity and continuity constraints for all the optical flows. For the latter, the fact that S-BVT Tx VCSELs are bound to a fixed wavelength, constrains/narrows the candidate set of feasible $F S$ for every optical flow. In other words, the available S-BVT VCSELs at the src determine the set of usable optical carriers (i.e., NCFs) for the optical flows's $F S$ s. Bearing this in mind, increasing the number of spatial paths to be considered by the RSA-CR algorithm (i.e., higher $K$ ) allows better dealing with the spectrum continuity and contiguity constraints for each required optical flow's $F S$. This is clearly reflected in the BBR performance as shown in Fig. 5. Considering the obtained BBR results for $K=1$, we observe that at low traffic load (i.e., HT=400s), the BBR is reduced (i.e., improved) around $47 \%, 48 \%$ and $52 \%$ when using RSA computing $K=3,6$ and 9 SPs, respectively. At high traffic load (i.e., HT=1200s), the improvement attained by $K=3,6$ and 9 when compared to $K=1$ is approximately $18 \%$, $24.5 \%$ and $25.4 \%$. Thus, as $K$ increases, the attained BBR improvement (with respect to $K=1$ ) does not proportionally grow. The rationale behind this is that increasing $K$ means to compute longer (in terms of hops and distance) candidate spatial paths. Using such longer spatial paths makes the RSA algorithm to rely on the lowest $O M$ for the S-BVT VCSELs. Thus, for a req, the number of required optical flows tends to be increased which entails not only requiring more resources to be allocated (at both S-BVT Tx and Rx) but also notably complicates the spectrum constraints for each optical flow.

Table III provides the average used S-BVT VCSELs and CO-Rxs for different HT and $K$ values. As discussed above, increasing the $K$ value enhances the obtained BBR metric, but also does increases the number of average used VCSELs and CO-Rxs. In other words, with higher $K$, available resources at the S-BVTs are more efficiently used, which leads to have 


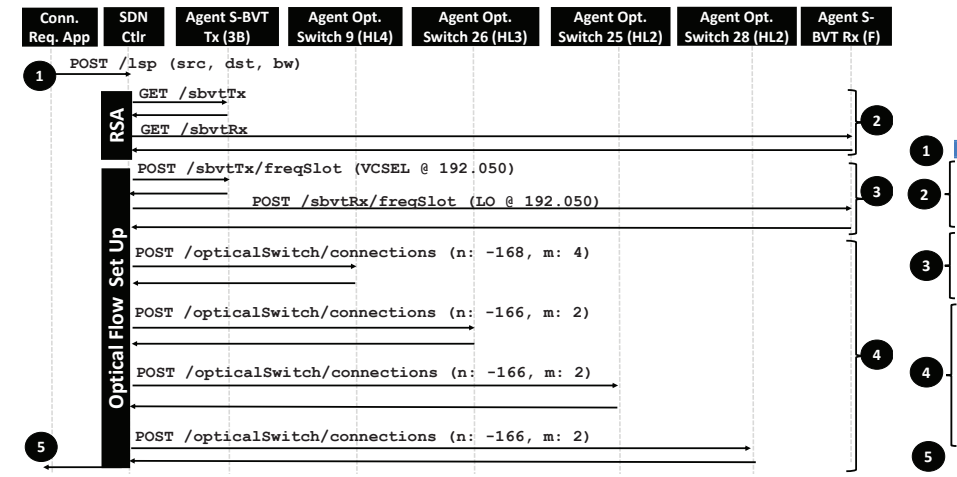

(a) Logical workflow

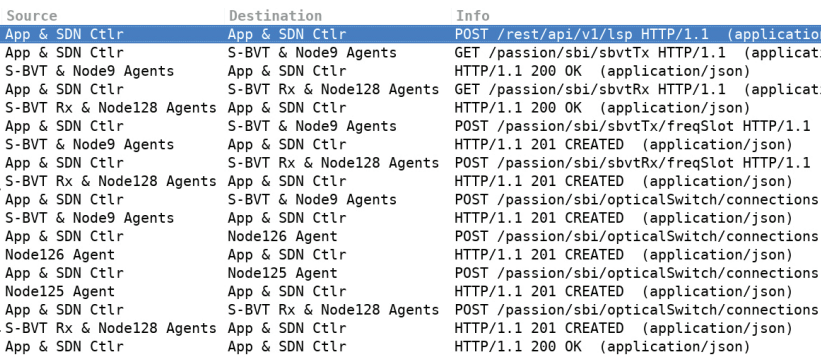

(b) REST API captured control messages

Fig. 3: Validation of the workflow among SDN controller - S-BVT and HL node agents.

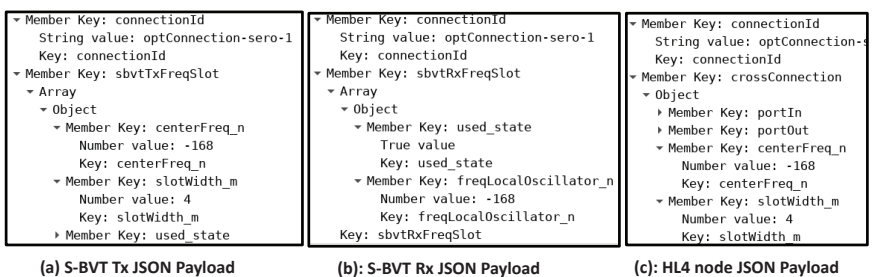

Fig. 4: REST API commands: JSON-encoded parameters.

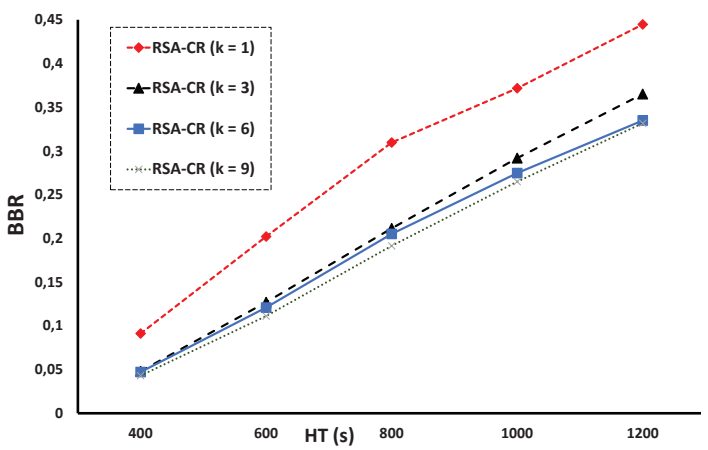

Fig. 5: Blocked Bandwidth Ratio (BBR) vs HT.

more connections co-existing within the network. However, this improvement in both BBR performance and S-BVT resource utilization is attained at the expenses of slightly increasing the average setup time as seen in Table III. That is, more candidate spatial paths are computed, which increases the RSA computation time.

\section{CONCLUSIONS}

To cope with the huge transport capacity needs expected in the metro network segment, an appealing approach is to exploit the benefits of photonic technologies. This leads to fulfil such transport capacity demands along with attaining efficient network cost, consumed energy, footprint, etc. The EC-H2020 PASSION project focuses on this metro network scenario by: i) designing and deploying programmable and modular SBVT devices leveraging the benefits of dense photonic integration and VCSEL technologies; ii) relying on a hierarchical flexi-grid optical metro infrastructure fostering the end-to-end optical flow aggregation and transport. We identify, deploy and validate the SDN controller functions and interfaces to dynamically compute paths and automatically program the PASSION transport layer. To this end, the capabilities and features of the adopted PASSION network technologies are taken on at the SDN controller. This results on designing and validating a control workflow along with RESTful APIs which are used, upon a new connection request, to compute and configure all the involved/selected metro network elements. Upon dynamic connection arrival, an on-line RSA algorithm based on a modified K-SP mechanism is devised. The RSA algorithm provides the computation of feasible spatial and spectral paths $(F S)$ for a number of optical flows required to satisfy the received req. To do that, the RSA considers different defined S-BVT VCSEL operations modes providing several data rates depending on the path distance and traversed number of hops. The RSA algorithm is assessed according to different figures of merit (e.g., BBR, average used of S-BVT VCSEL, etc.) along with supporting different K-SPs for a req.

\section{REFERENCES}

[1] CISCO White Paper, "Cisco Visual Networking Index: Forecast and Trends, 2017 - 2022 White Paper," Feb. 2019.

[2] M. Svaluto Moreolo, J. M. Fabrega, L. Nadal, R. Martínez and R. Casellas, "Synergy of Photonic Technologies and Software-Defined Networking in the Hyperconnectivity Era," IEEE J. of Lightwave Tech., vol. 37, no. 16, Aug. 2019.

[3] P. Paroli, A. Gatto and P. Boffi, "Long Wavelength VCSELs Exploitation for Low-Cost and Low-Power Consumption Metro and Access Network," in Proc. of 20th International Conference on Transparent Optical Networks (ICTON), July 2018.

[4] R. Martínez, et. al., "Proof-of-Concept Validation of SDN-Controlled VCSEL-based S-BVTs in Flexi-Grid Optical Metro Networks," in Proc. of Optical Fiber Communications (OFC), March 2019.

[5] EC H2020 PASSION Project, "http://www.passion-project.eu/wpcontent/uploads/2018/01/PASSION-Project-Fact-Sheet.pdf"

[6] Recommendantion ITU-T G.694.1, "Spectral grids for WDM applications: DWDM frequency grid", February 2012.

[7] R. Muñoz, et. al., "The ADRENALINE Testbed: An SDN/NFV Packet/Optical Transport Network and Edge/Core Cloud Platform for End-to-End 5G and IoT Services," In Proc., of European Conference on Networks and Communications (EuCNC), June 2017. 